\title{
N6-Methylandenosine-Related IncRNAs Play an Important Role in the Prognosis and Immune Microenvironment of Pancreatic Ductal Adenocarcinoma
}

\section{YuHai Hu}

Department of Hepatopancreatobiliary Surgery, Fujian Abdominal Surgery Research Institute, the First Affiliated Hospital of Fujian Medical University

YiPing Chen ( $\nabla$ cypgg@fjmu.edu.cn )

First Affiliated Hospital of Fujian Medical University

\section{Research}

Keywords: Pancreatic ductal adenocarcinoma, N6-methylandenosine, long non-coding RNA, prognostic signature, Immune

Posted Date: March 5th, 2021

DOI: https://doi.org/10.21203/rs.3.rs-274618/v1

License: (c) (1) This work is licensed under a Creative Commons Attribution 4.0 International License.

Read Full License 


\section{Abstract}

\section{Background}

Pancreatic ductal adenocarcinoma (PDAC) is a highly aggressive, fatal tumor. N6-methylandenosine $(\mathrm{m} 6 \mathrm{~A})$ methylation is the major epigenetic modification of RNA including IncRNAs. The roles of m6Arelated IncRNAs in PDAC have not been fully clarified. The aim of this study is to assess gene signatures and prognostic value of m6A-related IncRNAs in PDAC.

\section{Methods}

The Cancer Genome Atlas (TCGA) dataset and the International Cancer Genome Consortium (ICGC) dataset were explored to identify m6A-related IncRNAs. Univariate, least absolute shrinkage and selection operator (LASSO) and multivariate Cox regression were performed to construct the m6A-related IncRNAs prognostic riskscore (m6A-LPR) model to predict the overall survival (OS) in the TCGA training cohort. Kaplan-Meier curve with log-rank test and receiver operating characteristic (ROC) curve were used to evaluate the prognostic value of the m6A-LPR. Furthermore, the robustness of the m6A-LPR was further validated in the ICGC cohort. Tumor immunity was evaluated using ESTIMATE and CIBERSORT algorithms.

\section{Results}

A total of 262 m6A-related IncRNAs were identified in two datasets. In the TCGA training cohort, 28 prognostic m6A-related IncRNAs were identified and the m6A-LPR including four m6A-related IncRNAs was constructed. The m6A-LPR was able to identify high-risk patients with significantly poorer OS and accurately predict OS in both the TCGA training cohort and the ICGC validation cohort. Analysis of tumor immunity revealed that high-risk group had remarkably lower stromal, immune, and ESTIMATE scores. Moreover, high-risk group was associated with significantly higher levels of plasma B cells and resting NK cells infiltration, and lower levels of infiltrating resting memory CD4 T cells, monocytes and resting mast cells.

\section{Conclusions}

Our study proposed a robust m6A-related prognostic signature of IncRNAs for predicting OS in PDAC, which provides some clues for further studies focusing on the mechanism process underlying $\mathrm{m} 6 \mathrm{~A}$ modification of IncRNAs.

\section{Introduction}

Pancreatic ductal adenocarcinoma (PDAC) is a major histological subtype of pancreatic cancer. The incidence of PDAC is rising and the five-year survival rate is less than $5 \%$ with no significant improvement in survival over the past 10 years $[1,2]$. Surgical resection offers the only potentially curative treatment, but $80 \%$ of patients with PDAC are not amenable to surgery at diagnosis[3]. The efficacy of systemic 
treatment is limited and the advent of targeted and immune therapies are promising strategies to address this challenge. Thus, it is urgent to investigate potential therapeutic targets for PDAC.

N6-methylandenosine (m6A) RNA methylation is the main epigenetic modification of messenger RNAs (mRNAs) and non-coding RNAs (ncRNAs) [4], it has been confirmed to play critical regulatory roles in the modification of tumor RNAs[5]. M6A modifications are invertible and dynamical processes that are regulated by three kinds of $\mathrm{m} 6 \mathrm{~A}$ regulator, including methyltransferases ("writers"), signal transducers ("readers") and demethylases ("erasers")[6]. Recent studies demonstrated that m6A modification involves the regulation of oncogenesis and tumor progression in PDAC[7-12]. ALKBH5 serves as a PDAC suppressor by regulating the posttranscriptional activation of PER1 through m6A abolishment[7] and decreasing WIF-1 RNA methylation and mediating Wnt signaling[9]. Upregulation of METTL14 can promote the growth and metastasis of PDAC by decreasing of PERP levels[11].

Long non-coding RNAs (IncRNAs) regulate the biological functions of cells, including the proliferation, infiltration and metastasis of certain tumor cells[13], and dysregulation of IncRNAs had been reported to play a crucial role in the carcinogenicity of $\operatorname{PDAC}[8,14-16]$. A recent study found that m6A reader IGF2BP2 regulates IncRNA DANCR to promote cancer stemness-like properties and pancreatic cancer pathogenesis[8]. Nevertheless, the full impact of m6A regulators on the aberrant IncRNAs expression in cancers is still unclear and few studies have been conducted to investigate the mechanisms underlying how IncRNAs are regulated by m6A modification to involve in the onset and development of PDAC. Therefore, understanding how m6A modifications of IncRNAs contribute to PDAC progression can help to identify novel biomarkers as potential therapeutic targets.

In this study, The Cancer Genome Atlas (TCGA) dataset $(n=140)$ and the International Cancer Genome Consortium (ICGC) dataset $(n=63)$ were explored to identify 262 m6A-related IncRNAs in patients with PDAC. Then we found that 28 prognostic m6A-related IncRNAs in TCGA cohort and we constructed an m6A-related IncRNAs prognostic riskscore (m6A-LPR) model including four prognostic m6A-related IncRNAs to predict the overall survival (OS) of patients with PDAC. The relevance of the m6A-LPR with tumor immunity was also evaluated. Our results would be helpful to assess the prognosis of patients with PDAC and might offer the promise of individualized therapeutic interventions.

\section{Materials And Methods}

\section{Datasets, m6A-Related Genes and Annotation of IncRNAs}

For TCGA training dataset, normalized RNA sequencing data [Fragments Per Kilobase of transcript per Million mapped reads (FPKM) normalized] and the corresponding clinicopathological data of PDAC were obtained from the Genomic Data Commons Data Portal (https://portal.gdc.cancer.gov/). To obtain a ICGC validation dataset, normalized RNA-seq data of PACA-AU and the corresponding clinicopathological data were downloaded from the ICGC Data Portal (https://dcc.icgc.org). Only the patients with histological confirmed in PDAC was included, patients with OS $<30$ days or unknown OS status were excluded. Finally, we obtained the TCGA training cohort of 140 patients and the ICGC validation cohort of 
63 patients. Moreover, on the basis of published literatures, the expression data of 24 m6A-related genes were generated from the TCGA and ICGC datasets, including writers (METTL3, METTL14, METTL16, RBM15, RBM15B, WTAP, VIRMA [KIAA1429], CBLL1 and ZC3H13), erasers (ALKBH5 and FTO) and readers (YTHDF1, YTHDF2, YTHDF3, YTHDC1, YTHDC2, HNRNPC, HNRNPA2B1, IGF2BP1, IGF2BP2, IGF2BP3, $F M R 1, R B M X$ and $\angle R P P R C$ ). In our study, annotation of IncRNAs based on eight types of transcript (lincRNA, antisense, 3prime overlapping ncRNA, processed transcript, sense overlapping, sense intronic and macro IncRNA). Based on the Ensemble IDs and types of the transcript from the GENCODE website (https://www.gencodegenes.org/human/), 14830 IncRNAs were identified in the TCGA cohort and 12559 IncRNAs were identified in the ICGC cohort.

\section{Obtaining m6A-related IncRNAs}

Pearson correlation analyses were applied to explore m6A-related IncRNAs (|Pearson $\mathrm{R} \mid>0.4$ and $p<$ 0.001 ) in two cohorts. To ensure a more accurate corresponding relationship between m6A-related genes and IncRNAs, the results of correlation were built in "m6A-related gene/IncRNA/positive or negative correlation" format (eg. YTHDF1/ZFAS1/positive). The formatted results from the two cohorts were intersected to generate the 262 m6A-related IncRNAs from 582 correlations.

\section{Identifying prognostic m6A-related IncRNAs}

We selected OS as the endpoint. In TCGA training cohort, univariate Cox regression analysis was first conducted to identify the prognostic m6A-related IncRNAs, Next, least absolute shrinkage and selection operator (LASSO) Cox regression analysis was performed using the R package "glmnet" through 10-fold cross-validation. Finally, multivariate Cox regression analysis was conducted to identify the independent prognostic m6A-related IncRNAs. Based on the multivariate Cox regression result, a m6A-related IncRNA prognostic riskscore (m6A-LPR) model was developed for the PADC patients, each patient's risk score was calculated by a combination of the expression levels of IncRNAs and multivariate Cox regression coefficients in TCGA training cohort. We used the median risk score as the cut-off value to categorize the patients into high-risk group and low-risk group. The ICGC dataset were used as the validation cohort to verify the m6A-LPR. The time-dependent receiver operating characteristic (ROC) curves of the risk scores were conducted using the R package "timeROC" to evaluate the prognostic accuracy of m6A-LPR.

\section{Functional and pathway enrichment analysis}

In the TCGA cohort, based on the m6A-LPR, differentially expressed genes (DEGs) between the high-risk group and low-risk group were identified using the R package "limma" (|log2(Fold change)| $>1$ and False Discovery Rate $(F D R)<0.05)$. The 927 DEGs were obtained and imported into the "Metascape" website (https://metascape.org)[17] for functional and pathway enrichment analysis, including Reactome Gene Sets, Canonical Pathways, Gene Ontology (GO) Biological Processes and Kyoto Encyclopedia of Genes and Genomes Pathway (KEGG pathway).

\section{Tumor Immunity Analyses}


Stromal, immune, and estimate scores were calculated using the ESTIMATE algorithm[18] which was generated from the expression data in the TCGA dataset (https://bioinformatics.mdanderson.org/publicsoftware/estimate/), then we evaluated the differences in stromal, immune, and estimate scores between low- and high-risk group of PDAC patients. Furthermore, to infer the relative abundance of tumorinfiltrating immune cells, CIBERSORT deconvolution algorithm[19] was used, with the LM22 set representing 22 kinds of immune cell. We evaluated the differences in the immune infiltration of 22 immune cell subtypes between low- and high-risk group of PDAC patients.

\section{Potential drugs for prognostic m6A-related IncRNAs}

We used the Drug-LncRNA Module of the LncMAP database (http://bio-bigdata.hrbmu.edu.cn/LncMAP/) [20] to analyze the relationship between prognostic m6A-related IncRNAs and drugs, FDR less than 0.05 was considered significant. The m6A-related IncRNAs-drugs network was plotted by Cytoscape (version 3.8.2, http://www.cytoscape.org/)[21].

\section{Statistical analysis}

All statistical analyses and plots were performed using R Foundation Statistical software (version 4.0.2). Categorical variables were analyzed using $\chi 2$ test or Fisher's exact test. Continuous variables were analyzed using Student's t test or Wilcoxon test. Survival was estimated using the Kaplan-Meier survival curves and compared using the log-rank test. Univariate, LASSO and multivariate Cox regression analyses were performed to identify independent prognostic m6A-related IncRNAs and to develop the m6A-LPR. The hazard ratio $(\mathrm{HR})$ and $95 \%$ confidence interval $(\mathrm{Cl})$ were calculated. Unless otherwise stipulated, two tailed $p<0.05$ was considered statistically significant.

\section{Results}

\section{Identification of m6A-Related IncRNAs in PDAC Patients}

The work flow was shown in Fig. 1. Cumulatively, 140 PDAC patients from TCGA cohort and 63 PDAC patients from ICGC cohort were included in our study; for whom, the baseline clinical features were presented in Additional file 1: Table S1. Firstly, we identified 14830 IncRNAs in the TCGA cohort and 12559 IncRNAs in the ICGC cohort. Next, we extracted the expression data of IncRNAs and 24 m6Arelated genes from the TCGA and the ICGC cohorts. Pearson correlation analyses were performed to identify m6A-related IncRNAs in two cohorts ( $\mid$ Pearson $R \mid>0.4$ and $p<0.001)$. We obtained the 2672 correlations in the TCGA cohort and 21008 correlations in ICGC cohorts, then the corresponding relationship between m6A-related genes and IncRNAs in two cohorts were intersected. Finally, 585 shared correlations were obtained in both two cohorts, then 262 m6A-related IncRNAs were extracted from the shared correlations.

\section{Identification of prognostic m6A-Related IncRNAs}


Univariate Cox regression was applied to identify prognostic m6A-related IncRNAs from the $262 \mathrm{~m} 6 \mathrm{~A}$ related IncRNAs in the TCGA traing cohort $(p<0.05)$. The result showed that 28 m6A-related IncRNAs were significantly associated with the OS (Fig. 2A), including 2 risky IncRNAs and 26 protective IncRNAs, and the correlations between the 28 IncRNAs and the m6A-related genes in the TCGA cohort are shown in Fig. 2B.

\section{Establishment of the m6A-LPR in the TCGA Training Cohort}

To construct the m6A-LPR for predicting the OS of PDAC patients, LASSO Cox analysis was performed to the 28 prognostic m6A-related IncRNAs in the TCGA cohort and 12 m6A-related IncRNAs were screened (Fig. 3A). Next, multivariate Cox proportional regression analysis was performed for analyzing these 12 m6A-related IncRNAs in TCGA cohort to construct the m6A-LPR. The m6A-LPR comprising four m6Arelated IncRNAs, including MIR4435-1HG, RP5-1112D6.4, RP11-582J16.5 and RP11-999E24.3, was developed through the summary of the expression values of these four m6A-related IncRNAs multiplied by corresponding coefficients derived from the above multivariable Cox regression analysis (Fig. 3B, C). The downregulated RP5-1112D6.4, RP11-582J16.5, and RP11-999E24.3 with HR $<1$ were considered to be tumor suppressors, while the upregulated MIR4435-1HG with $\mathrm{HR}>1$ was considered to be oncogenes. The Kaplan-Meier survival curves showed that higher expression of RP5-1112D6.4, RP11-582J16.5 and RP11-999E24.3 and lower expression of MIR4435-1HG were correlated with impoved OS in the TCGA cohort (Fig. 3D-G). The expression of four m6A-related IncRNAs were also associated with the clinicopathological and immune signatures of PDAC, such as WHO grade, TP53 mutation status, KRAS mutation status, ESTIMATE score, immune score, stromal score, and tumor mutation burden (TMB) (Fig. $3 \mathrm{H})$. The genomic information of these four m6A-related IncRNAs and the corresponding correlation with m6A regulators were presented in Table 1. The risk score for each patient was calculated by following formula based on m6A-LPR: [(0.205)*expression value of MIR4435-1 HG] - [(0.525)*expression value of RP11-582J16.5] - [(0.658)*expression value of RP11-999E24.3] - [(0.275)*expression value of RP5-

1112D6.4]. Based on the median value of risk scores, patients were categorized into low-risk and high-risk groups. Kaplan-Meier survival curves showed that PDAC patients with lower risk scores had better OS ( $p$ $=0.0011)$ (Fig. 4A). Survival status and risk score distributions were illustrated in Fig. 4B. The ROC curves demonstrated that m6A-LPR had a good performance for predicting OS in the TCGA cohort (1-year AUC $=$ 0.760, 2-year AUC = 0.722; Fig. 4C).

\section{Validation of the m6A-LPR in the ICGC Cohort}

In order to further validate the robustness of m6A-LPR, the risk scores were calculated in the ICGC cohort using the above equation. Based on the median risk score, PDAC patients in the ICGC cohort were also divided into low- and high-risk subgroups. Consistent with the results in the TCGA training cohort: PDAC patients with lower risk scores had better OS in the ICGC validation cohort $(p=0.020)$ (Fig. 4D). Survival status and risk score distributions were shown in Fig. 4E. The ROC curves also demonstrated that m6ALPR had a prognostic value for PDAC patients in the ICGC cohort (1-year AUC $=0.657$, 2-year AUC $=0.729$; 
Fig. 4F). These results showed that the m6A-LPR based prognostic signature had a robust and stable ability in prognosis prediction for PDAC.

\section{Principal Component Analysis}

Principal component analysis (PCA) was applied to evaluate the discrepancies between the low- and high-risk subgroups based on the expression of the four m6A-related IncRNAs in m6A-LPR (Fig. 5A, B). The results showed that the samples screened by the four m6A-related IncRNAs could clearly divide the whole patients into a low-risk and high-risk group in both the TCGA and ICGC cohorts.

\section{Stratification Analysis of the m6A-LPR in clinicopathological features}

PDAC patients with WHO grade III-IV, mutant TP53 and mutant KRAS (Fig. 6A-C) had higher risk scores, whereas the risk scores were not correlated with stage, $T$ category and $\mathrm{N}$ category (Fig. 6D-F). To evaluate whether m6A-LPR was an independent prognostic factor for PDAC patients, univariate and multivariate Cox analyses were performed. In the TCGA cohort, univariate Cox analysis showed that m6A-LPR was significantly associated with OS (HR: $2.72,95 \% \mathrm{Cl}: 1.94-3.81, \mathrm{p}<0.001)$ and multivariate Cox analysis indicated that m6A-LPR was an independent predictor of OS (HR: 2.77, 95\% Cl: 1.93-3.96, p < 0.001; Fig. $6 G, H)$. In the ICGC validation cohort, univariate and multivariate Cox analyses also indicated that m6ALPR was an independent predictor of OS for PDAC patients (univariate: HR: 1.83, 95\% Cl: 1.12-2.66, p = 0.012; multivariate: HR: $1.75,95 \% \mathrm{Cl}: 1.12-2.50, p=0.020$; Fig. $6 \mathrm{I}, \mathrm{J})$. These results demonstrated that m6A-LPR might be helpful for clinical prognosis evaluation as an independent prognostic indicator.

\section{Stratification Analysis of the m6A-LPR in immune features}

The relationship between m6A-LPR and tumor immunity was further evaluated. Tumor purity, TMB, Immune Checkpoint Molecules and the infiltration level of immune cell were estimated. PDAC patients in the high-risk group had remarkably lower stromal, immune, and ESTIMATE scores, indicating a lower level of stroma, immune cell infiltration, and tumor purity (Fig. 7A). Furthermore, PDAC patients in the high-risk group had significantly higher levels of TMB (Fig. 7B) and lower levels of CTLA4 expression (Fig. 7C). To further investigate the underlying molecular mechanisms of the m6A-LPR and its relevance to tumor immunity, the relative abundance of 22 tumor-infiltrating immune cells was assessed for each patient using CIBERSORT. The high-risk group was associated with significantly higher levels of plasma B cells and resting NK cells infiltration, and lower levels of infiltrating resting memory CD4 T cells, monocytes and resting mast cells (Fig. 7D).

\section{Functional and pathway enrichment analysis}

To explore the potential biological processes and pathways of the molecular discrepancy between the low-risk and high-risk groups, 927 differential expression genes (DEGs) were identified between the lowrisk and high-risk groups in the TCGA cohort (|log2 (fold change) $\mid>1$ and $p<0.05$ ). Functional and pathway enrichment analysis indicated these DEGs were mainly enriched in these aspects: digestion, neuronal system and peptide hormone metabolism (Reactome Gene Sets); NABA matrisome-associated 
(Canonical Pathways); pancreatic secretion, neuroactive ligand-receptor interaction and cytokine-cytokine receptor interaction (KEGG Pathways); regulation of ion transport, chemical synaptic transmission, regulation of system process, signal release, neuropeptide signaling pathway and second-messengermediate signaling (GO Biological Processes) (Fig. 8A-C). These results could give us some insights into the potential molecular mechanisms of the m6A-LPR.

\section{Explore Potential Drugs that have a Therapeutic Effect on PDAC}

From the Drug-LncRNA Module of the LncMAP database, we obtained 304304 drug-IncRNA interaction pairs. A total of 28 prognostic m6A-related IncRNAs were then imported into the database to predict the potential drugs of the genes, and 75 drug-IncRNA interactions were extracted when FDR $<0.05$. The network including 18 prognostic m6A-related IncRNAs and 18 drugs was identified (Fig. 9). The five most interaction with prognostic m6A-related IncRNAs drugs were Panobinostat, L-685458, Palbociclib, Crizotinib and TAE684.

\section{Discussion}

PDAC is an extremely challenging disease, since only $80 \%$ of patients with PDAC are not amenable to surgery at diagnosis[3]. Recently, with the benefit of high-throughput sequencing, studies to explore the molecular markers of PDAC at the molecular and cellular level have got breakthroughs, which will be helpful for increasing the prognostic accuracy and introducing potential therapeutic targets for PDAC. An increasing number of studies indicated that epigenetic alterations can largely effect cancer progression[22]. Among that, m6A modification is the most common epigenetic methylated modification of mRNAs and ncRNAs[4], it has been confirmed to play critical regulatory roles in the modification of tumor RNAs[5]. Aberrant IncRNAs had been found to be a important role in the carcinogenicity of PDAC[8, 14-16]. However, few studies have been conducted to investigate the mechanisms underlying how IncRNAs are regulated by m6A modification to involve in the onset and development of PDAC Therefore, we tried to identify m6A-related IncRNAs through bioinformatics analysis from two public datasets. Twenty-eight m6A-related IncRNAs had prognostic value, and four of them were screened to bulid an m6A-LPR for predicting the OS of PDAC patients. Furthermore, we explored the correlation of m6A-LPR with clinicopathological and immune features of PDAC and tried to find potential target drugs for prognostic m6A-related IncRNAs.

Previous studies had shown that the stability of IncRNAs is enhanced by the accumulation of m6A modifications[23], with the binding of low-complexity proteins[24], interactions with m6A readers[8], and additional regulatory mechanisms. Recent studies had demonstrated that m6A modification can regulate oncogenesis and tumor progression in PDAC[7-12], but it is still unclear how m6A modification affects the occurrence and progression of PDAC in a IncRNA-dependent pattern. The m6A eraser ALKBH5 could demethylate the IncRNA KCNK15-AS1 and inhibit KCNK15-AS1-mediated pancreatic cancer cell motility[25]. The m6A reader IGF2BP2 could interact with the IncRNA DANCR and promote cancer stemness-like properties and pancreatic cancer pathogenesis[8]. Studies had shown that m6A 
modification of IncRNAs may have an effect on the occurrence and progression of cancer and IncRNAs may act as target for m6A regulators to influence aggressive tumor progression. Based on these evidences, we should pay more attention to the interactions between IncRNAs and m6A modifications in order to identify potential therapeutic targets or prognosis markers of cancers.

We identified 28 prognostic m6A-related IncRNAs from TCGA dataset, and four of them were included in the m6A-LPR and validated in ICGC dataset. RP5-1112D6.4, RP11-582J16.5, and RP11-999E24.3 were protective genes, MIR4435-1 HG was risky gene. MIR4435-1 HG is highly expressed and acts as a risky gene in renal cell carcinoma, and it promotes cell proliferation, migration and invasive capacity of renal carcinoma cells[26]. The other three IncRNAs have not been reported in the literatures and the functions of them are unknown. We performed functional analysis of the DEGs in low- and high-risk patients stratified by m6A-LPR to explore the role of four m6A-related IncRNAs in PDAC. The analysis revealed that four m6A-related IncRNAs were significantly enriched in cell ion exchange (regulation of ion transport, inorganic ion homeostasis and anion transport) and biological processes of signaling pathways (neuroactive ligand-receptor interaction, signal release, cytokine-cytokine receptor interaction, neuropeptide signaling pathway and second-messenger-mediated signaling). The result implied that the m6A-LPR might be related to maintain cellular homeostasis and cell injury, thus affecting the progression of the tumor. The findings are probably used to develop new targeted anti-cancer therapies for PDAC if the hypothesis can be proved.

In many types of tumors, such as skin melanoma, breast cancer, colon cancer and non-small cell lung cancer, tumor immune infiltrating cells account for a high proportion based on IncRNA sequencing data[27]. The IncRNA Inc-EGFR can stimulate T-regulatory cells differentiation thus promoting hepatocellular carcinoma immune evasion[28]. LINC00473 can drive the progression of pancreatic cancer via upregulating PD-L1[29]. We believe that IncRNAs are the key to tumor immunotherapy. Therefore, we explored the relationship between m6A-LPR and tumor immunity, and we found that m6A-LPR for PDAC was associated with TMB, tumor purity, and the infiltration of immune cell subtypes.

This study included two PDAC datasets, the TCGA and ICGC datasets, and m6A-LPR containing four prognostic m6A-related IncRNAs were built in the TCGA dataset and validated in the ICGC dataset, but there were several limitations in our study. First, the interactions between IncRNAs and m6A-related genes were obtained from two datasets in our study, them should be confirmed through in vivo and in vitro experiments. Second, in two datasets, the clinical data are incomplete and exist selection bias. Third, traditional statistical analyzes were used to construct and validate the prognostic risk model of m6A-LPR. Although these methods had been utilized and validated in many studies, it is crucial to improve further studies with more advanced methodologies. To further verify our bioinformatics results, in-depth studies on the m6A-LPR containing four prognostic m6A-related IncRNAs, including molecular mechanisms and functional experiments, are needed.

\section{Conclusion}


In summary, we built and validated a prognostic model of m6A-LPR containing four prognostic m6Arelated IncRNAs. The m6A-LPR not only provides additional information for PDAC prognostic analyses, but also affects the immunity of PDAC. Further studies are needed to validate our model and to explore the molecular mechanism and function of m6A-LPR in the regulation of anti-tumor immunity, our results may provide some clues for further studies.

\section{Abbreviations}

PDAC: Pancreatic ductal adenocarcinoma; m6A: N6-methylandenosine; TCGA: The Cancer Genome Atlas; ICGC: International Cancer Genome Consortium; LASSO: least absolute shrinkage and selection operator; m6A-LPR: m6A-related IncRNAs prognostic riskscore; OS: overall survival; ROC: receiver operating characteristic; mRNAs: messenger RNAs; IncRNAs: Long non-coding RNAs; FPKM: Fragments Per Kilobase of transcript per Million; DEGs: differentially expressed genes; FDR: False Discovery Rate; GO: Gene Ontology; KEGG: Kyoto Encyclopedia of Genes and Genomes; HR: hazard ratio; Cl: confidence interval; TMB: tumor mutation burden.

\section{Declarations}

\section{Acknowledgements}

Not applicable.

\section{Authors' contributions}

YuHai Hu performed the collection, assembly and interpretation of data, edited the manuscript; YiPing Chen wrote the manuscript, designed the study and confirmed the data presented in the manuscript. All authors read and approved the final manuscript.

\section{Funding}

Not applicable.

\section{Availability of data and materials}

TCGA training data was downloaded from the TCGA database (http://cancergenome.nih.gov) under the accession number TCGA-PAAD. ICGC validation data was downloaded from the ICGC database (https://dcc.icgc.org) under the accession number PACA-AU.

\section{Ethics approval and consent to participate}

Not applicable.

\section{Consent for publication}


Not applicable.

\section{Competing interests}

The authors declare that they have no competing interests.

\section{References}

1. Quaresma M, Coleman MP, Rachet B: 40-year trends in an index of survival for all cancers combined and survival adjusted for age and sex for each cancer in England and Wales, 1971-2011: a population-based study. Lancet 2015, 385:1206-1218.

2. Bates SE: Pancreatic Cancer: Challenge and Inspiration.Clin Cancer Res 2017, 23:1628.

3. Strobel O, Neoptolemos J, Jäger D, Büchler MW: Optimizing the outcomes of pancreatic cancer surgery.Nat Rev Clin Oncol 2019, 16:11-26.

4. Fu Y, Dominissini $D$, Rechavi G, He C: Gene expression regulation mediated through reversible $\mathrm{m} \otimes \mathrm{A}$ RNA methylation. Nat Rev Genet 2014, 15:293-306.

5. Pan Y, Ma P, Liu Y, Li W, Shu Y: Multiple functions of m(6)A RNA methylation in cancer.J Hematol Oncol 2018, 11:48.

6. Zaccara S, Ries RJ, Jaffrey SR: Reading, writing and erasing mRNA methylation. Nat Rev Mol Cell Biol 2019, 20:608-624.

7. Guo X, Li K, Jiang W, Hu Y, Xiao W, Huang Y, Feng Y, Pan Q, Wan R: RNA demethylase ALKBH5 prevents pancreatic cancer progression by posttranscriptional activation of PER1 in an m6AYTHDF2-dependent manner.Mol Cancer 2020, 19:91.

8. Hu X, Peng WX, Zhou H, Jiang J, Zhou X, Huang D, Mo YY, Yang L: IGF2BP2 regulates DANCR by serving as an N6-methyladenosine reader.Cell Death Differ 2020, 27:1782-1794.

9. Tang $B$, Yang $Y$, Kang $M$, Wang $Y$, Wang $Y, B i$ Y, He S, Shimamoto F: m(6)A demethylase ALKBH5 inhibits pancreatic cancer tumorigenesis by decreasing WIF-1 RNA methylation and mediating Wnt signaling.Mol Cancer 2020, 19:3.

10. Tian J, Zhu Y, Rao M, Cai Y, Lu Z, Zou D, Peng X, Ying P, Zhang M, Niu S, et al: N(6)-methyladenosine mRNA methylation of PIK3CB regulates AKT signalling to promote PTEN-deficient pancreatic cancer progression. Gut 2020, 69:2180-2192.

11. Wang M, Liu J, Zhao Y, He R, Xu X, Guo X, Li X, Xu S, Miao J, Guo J, et al: Upregulation of METTL14 mediates the elevation of PERP mRNA N(6) adenosine methylation promoting the growth and metastasis of pancreatic cancer.Mol Cancer 2020, 19:130.

12. Zhang J, Bai R, Li M, Ye H, Wu C, Wang C, Li S, Tan L, Mai D, Li G, et al: Excessive miR-25-3p maturation via N(6)-methyladenosine stimulated by cigarette smoke promotes pancreatic cancer progression. Nat Commun 2019, 10:1858.

13. Schmitt AM, Chang HY: Long Noncoding RNAs in Cancer Pathways. Cancer Cell 2016, 29:452-463. 
14. Li ZH, Zhao XH, Zhou Y, Liu YM, Zhou QB, Ye HL, Wang YX, Zeng JL, Song YD, Gao WC, et al: The long non-coding RNA HOTTIP promotes progression and gemcitabine resistance by regulating HOXA13 in pancreatic cancer. Journal Of Translational Medicine 2015, 13.

15. Ma CC, Nong KT, Zhu HD, Wang WW, Huang XY, Yuan Z, Ai KX: H19 promotes pancreatic cancer metastasis by derepressing let-7's suppression on its target HMGA2-mediated EMT. Tumor Biology 2014, 35:9163-9169.

16. Pang EJ, Yang R, Fu XB, Liu YF: Overexpression of long non-coding RNA MALAT1 is correlated with clinical progression and unfavorable prognosis in pancreatic cancer. Tumor Biology 2015, 36:24032407.

17. Zhou Y, Zhou B, Pache L, Chang M, Khodabakhshi AH, Tanaseichuk O, Benner C, Chanda SK: Metascape provides a biologist-oriented resource for the analysis of systems-level datasets. Nat Commun 2019, 10:1523.

18. Yoshihara K, Shahmoradgoli M, Martínez E, Vegesna R, Kim H, Torres-Garcia W, Treviño V, Shen H, Laird PW, Levine DA, et al: Inferring tumour purity and stromal and immune cell admixture from expression data.Nat Commun 2013, 4:2612.

19. Gentles AJ, Newman AM, Liu CL, Bratman SV, Feng W, Kim D, Nair VS, Xu Y, Khuong A, Hoang CD, et al: The prognostic landscape of genes and infiltrating immune cells across human cancers. Nat Med 2015, 21:938-945.

20. Li Y, Li L, Wang Z, Pan T, Sahni N, Jin X, Wang G, Li J, Zheng X, Zhang Y, et al: LncMAP: Pan-cancer atlas of long noncoding RNA-mediated transcriptional network perturbations.Nucleic Acids Res 2018, 46:1113-1123.

21. Shannon P, Markiel A, Ozier O, Baliga NS, Wang JT, Ramage D, Amin N, Schwikowski B, Ideker T: Cytoscape: a software environment for integrated models of biomolecular interaction networks. Genome Res 2003, 13:2498-2504.

22. Ilango S, Paital B, Jayachandran P, Padma PR, Nirmaladevi R: Epigenetic alterations in cancer.Front Biosci (Landmark Ed) 2020, 25:1058-1109.

23. Ni W, Yao S, Zhou Y, Liu Y, Huang P, Zhou A, Liu J, Che L, Li J: Long noncoding RNA GAS5 inhibits progression of colorectal cancer by interacting with and triggering YAP phosphorylation and degradation and is negatively regulated by the m(6)A reader YTHDF3.Mol Cancer 2019, 18:143.

24. Hazra D, Chapat C, Graille M: m『A mRNA Destiny: Chained to the rhYTHm by the YTH-Containing Proteins. Genes (Basel) 2019, 10.

25. He Y, Hu H, Wang Y, Yuan H, Lu Z, Wu P, Liu D, Tian L, Yin J, Jiang K, Miao Y: ALKBH5 Inhibits Pancreatic Cancer Motility by Decreasing Long Non-Coding RNA KCNK15-AS1 Methylation.Cell Physiol Biochem 2018, 48:838-846.

26. Wu K, Hu L, Lv X, Chen J, Yan Z, Jiang J, Cheng Y, Hou J: Long non-coding RNA MIR4435-1 HG promotes cancer growth in clear cell renal cell carcinoma.Cancer Biomark 2020, 29:39-50.

27. Yu WD, Wang H, He QF, Xu Y, Wang XC: Long noncoding RNAs in cancer-immunity cycle.J Cell Physiol 2018, 233:6518-6523. 
28. Jiang R, Tang J, Chen Y, Deng L, Ji J, Xie Y, Wang K, Jia W, Chu WM, Sun B: The long noncoding RNA Inc-EGFR stimulates T-regulatory cells differentiation thus promoting hepatocellular carcinoma immune evasion. Nat Commun 2017, 8:15129.

29. Zhou WY, Zhang MM, Liu C, Kang Y, Wang JO, Yang XH: Long noncoding RNA LINC00473 drives the progression of pancreatic cancer via upregulating programmed death-ligand 1 by sponging microRNA-195-5p.J Cell Physiol 2019, 234:23176-23189.

\section{Tables}

Due to technical limitations, table 1 is only available as a download in the Supplemental Files section.

\section{Figures}




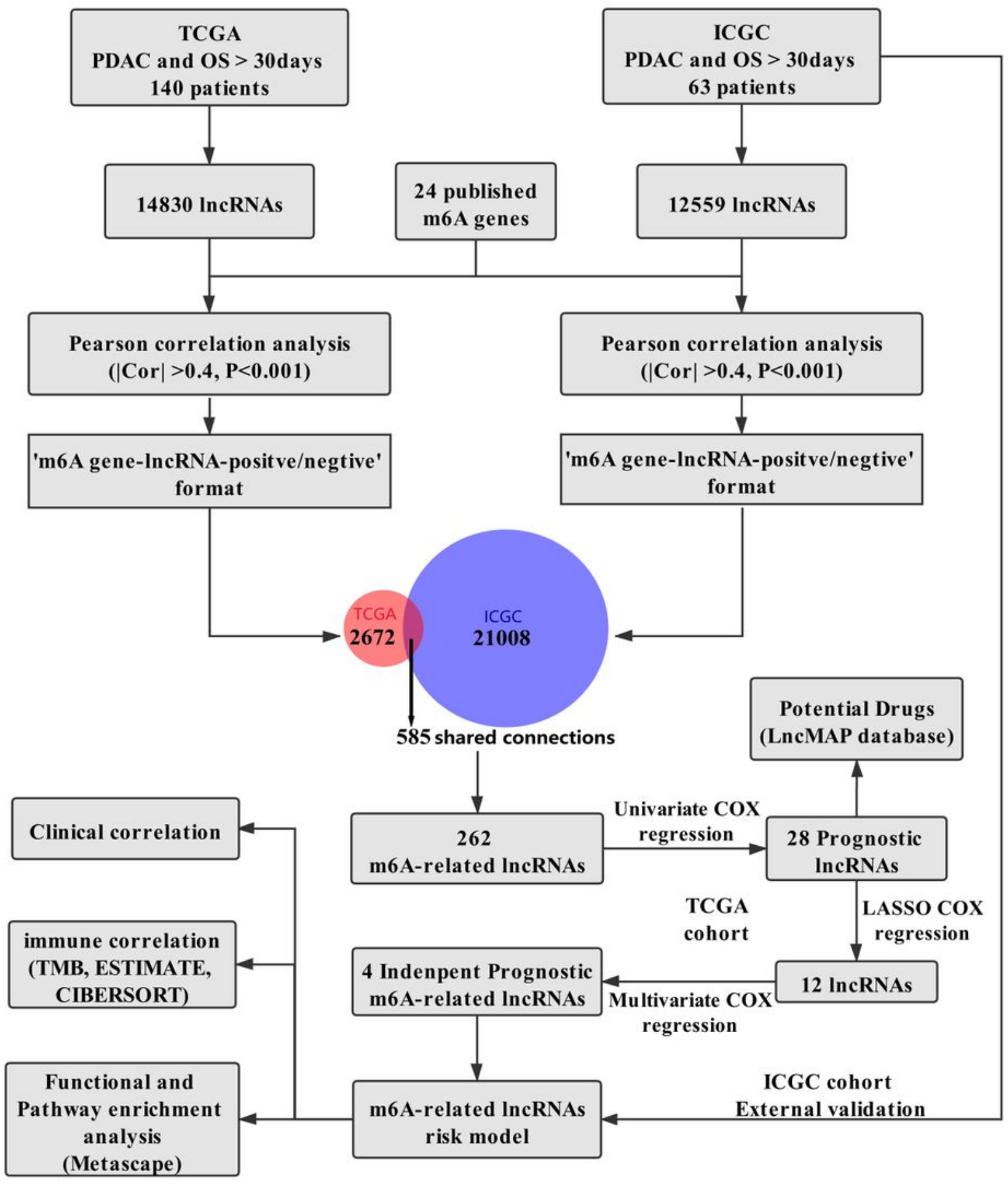

Figure 1

Flow chart of this study. 
A

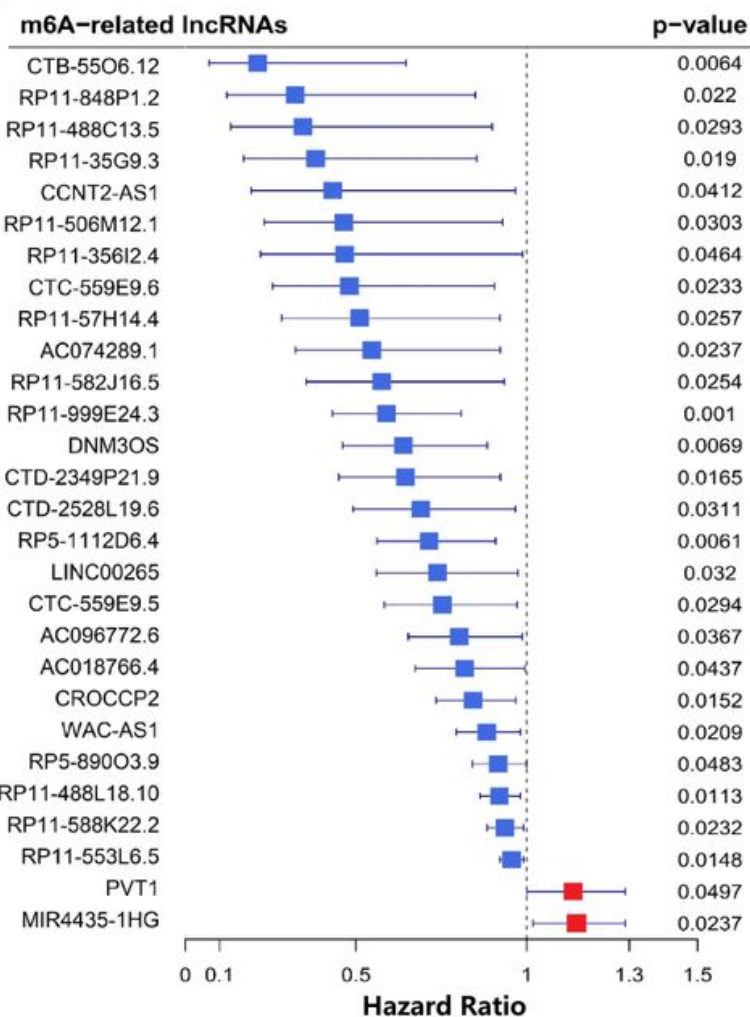

B

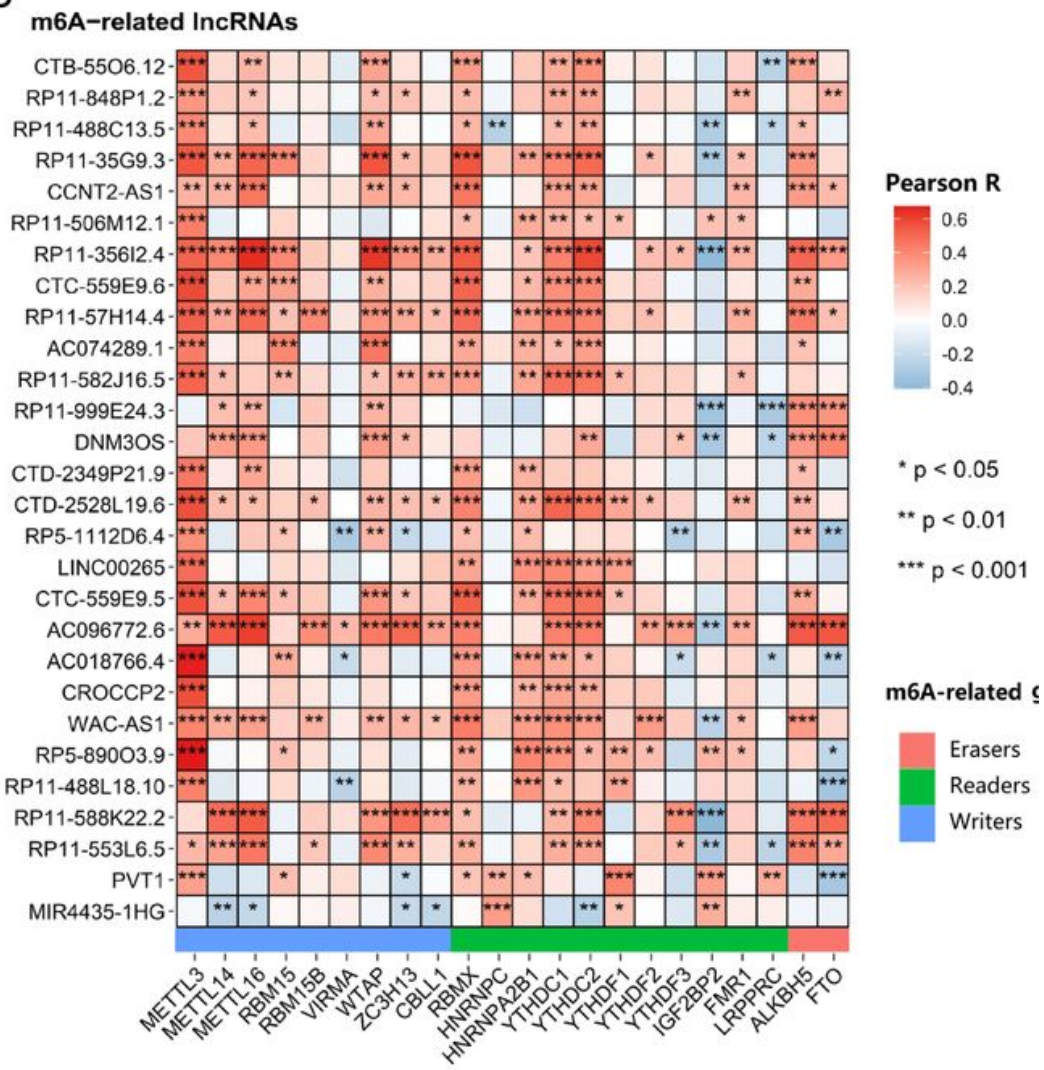

\section{Figure 2}

A The twenty-eight prognostic m6A-related IncRNAs in The Cancer Genome Atlas (TCGA) cohort. B Heatmap of the correlations between m6A-related genes and the 28 prognostic m6A-related IncRNAs. 
A

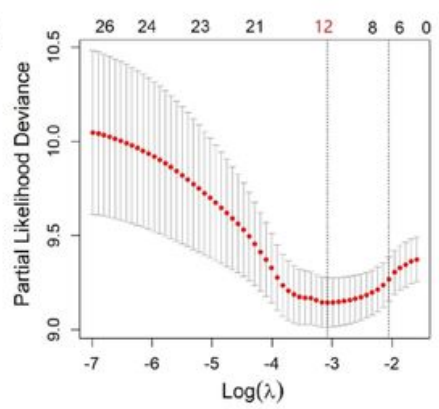

D

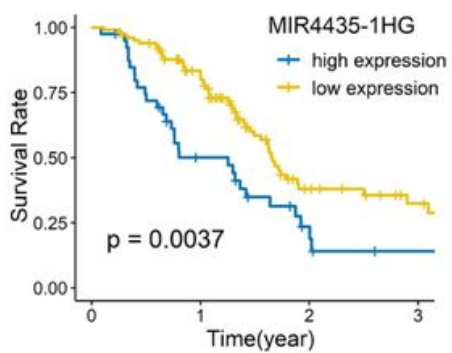

$\mathbf{F}$

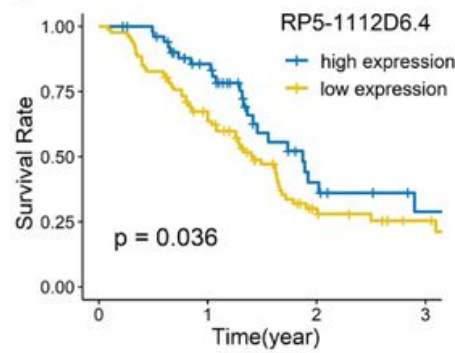

B

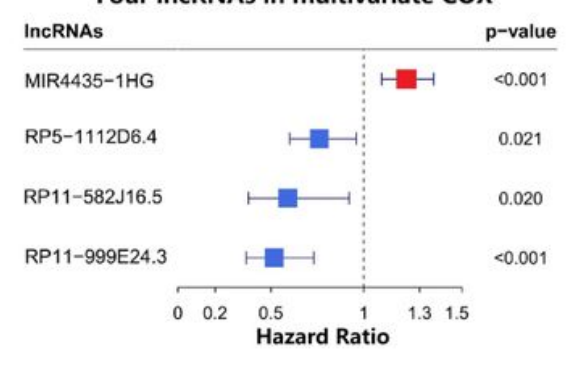

C

Coefficients of IncRNAs in risk model

H

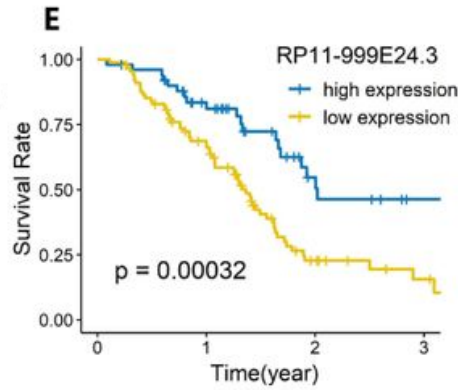

\section{G}

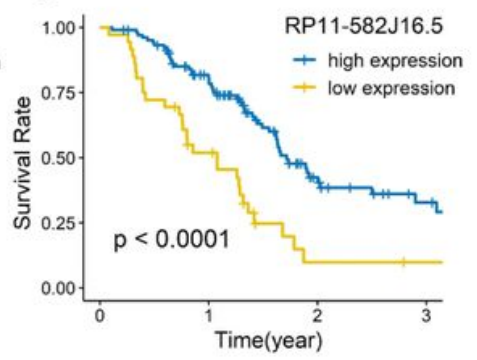

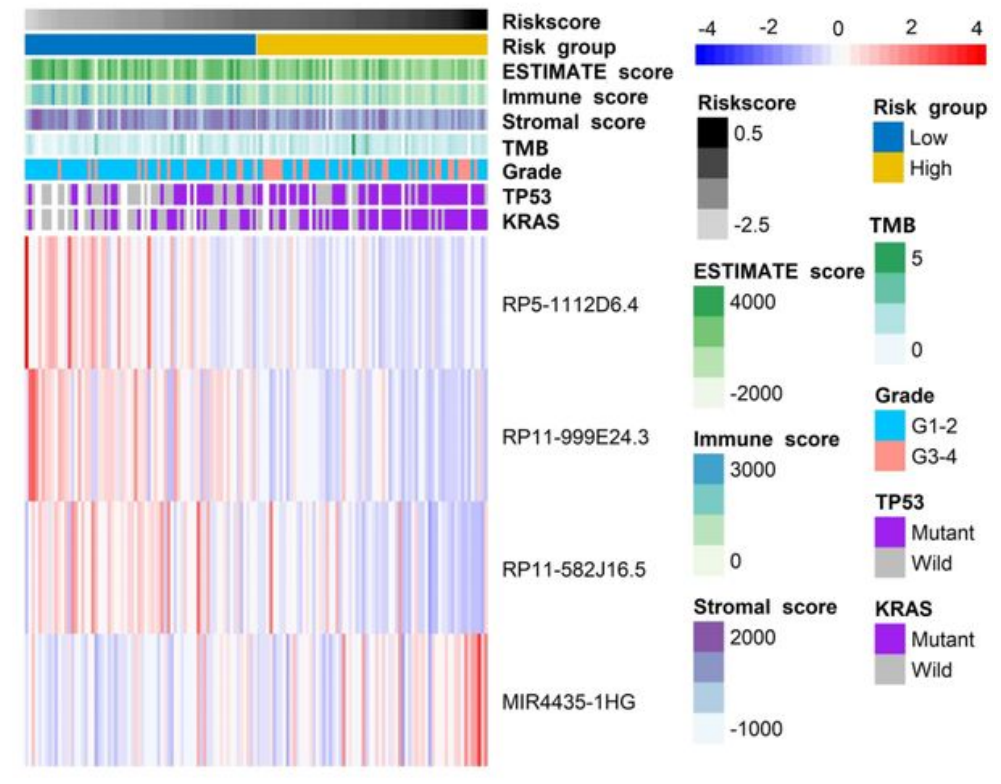

Figure 3

A Least absolute shrinkage and selection operator (LASSO) regression was performed, calculating the minimum criteria. B-C Multivariate cox regression was performed, calculating the hazard ratio (HR), $p$ value (B) and and coefficients (C) for four m6A-related IncRNAs in risk model. D-G Kaplan-Meier curves showing that patients with different expression levels of the four m6A-related IncRNAs had different overall survival. $\mathrm{H}$ Heatmap of the associations between the expression levels of the four m6A-related IncRNAs and clinicopathological features in The Cancer Genome Atlas (TCGA) cohort. 

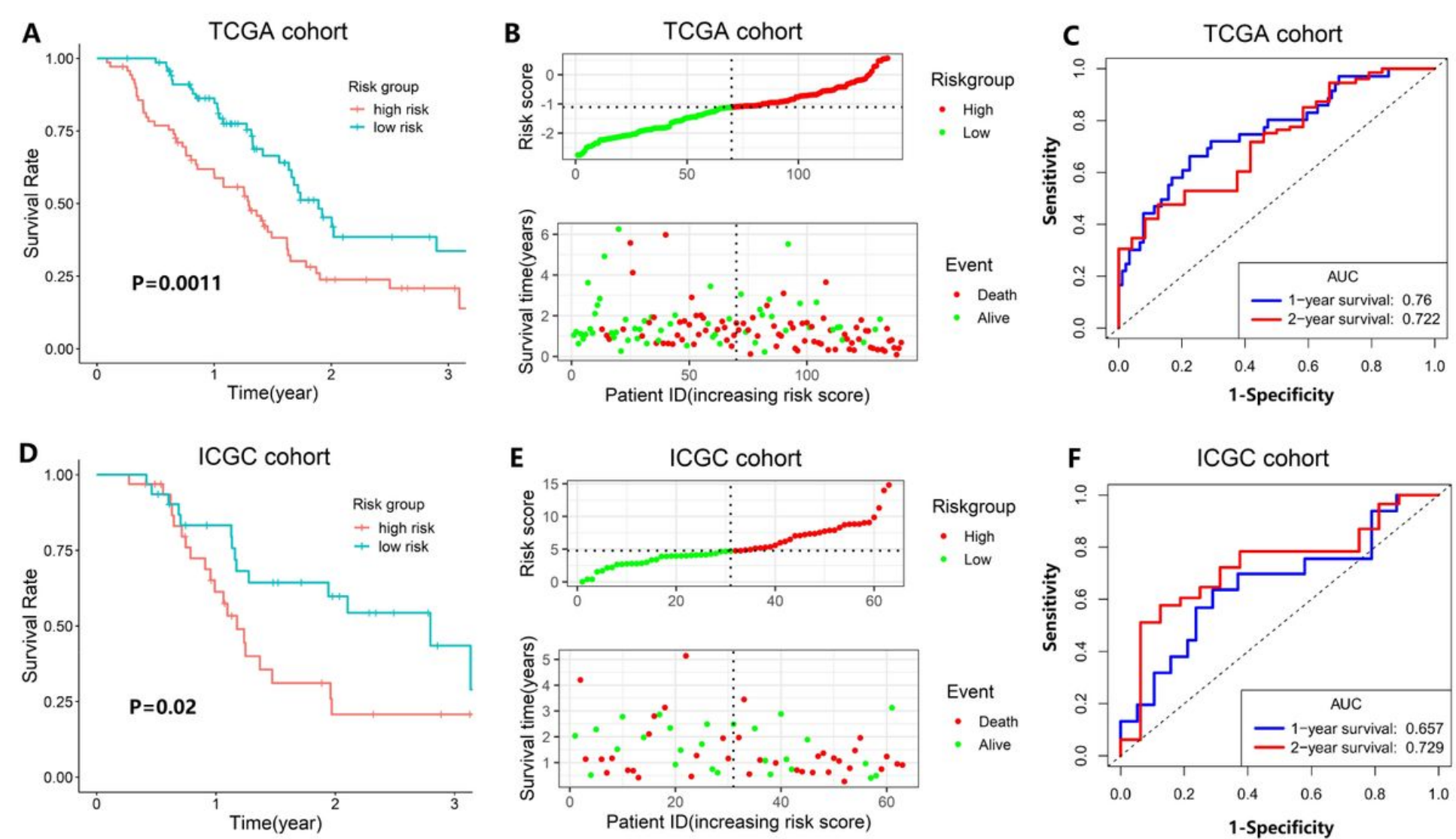

Figure 4

A Kaplan-Meier curves showed that the high-risk group had worse overall survival than the low-risk group in the training cohort. B Distributions of risk scores based on the m6A-related IncRNA prognostic riskscore (m6A-LPR) and survival status of patients in the training cohort. C Receiver operating characteristic (ROC) curves of m6A-LPR for predicting the 1/2-year survival in the training cohort. D Kaplan-Meier curves showing that the high-risk group had worse overall survival than the low-risk group in the validation cohort. E Distributions of risk scores and survival status of patients in the validation cohort. F ROC curves of m6A-LPR for predicting 1/2-year survival in the validation cohort. 

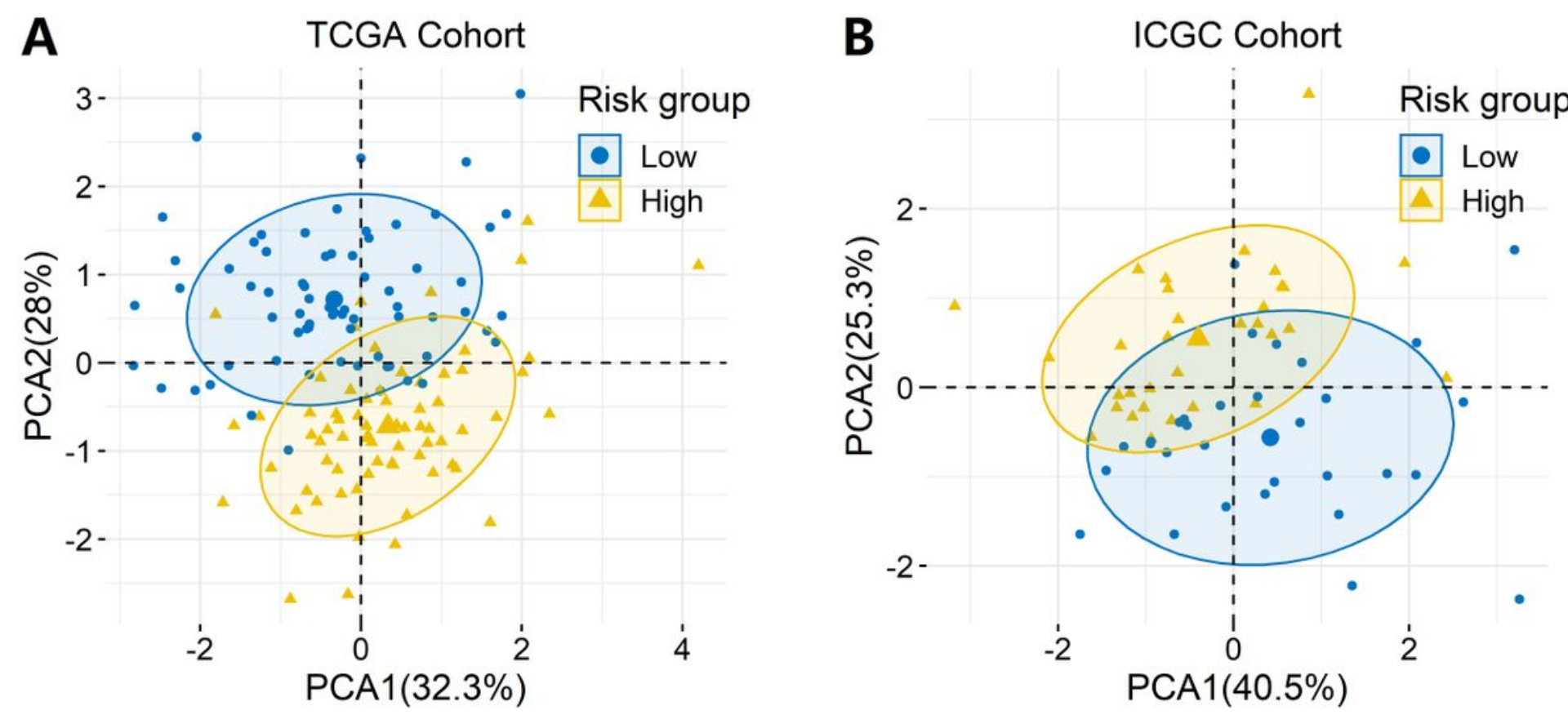

\section{Figure 5}

Principal component analysis (PCA) between the low- and high-risk groups based on the expression of the four m6A-related IncRNAs in m6A-LPR in the training cohort and the validation cohort.
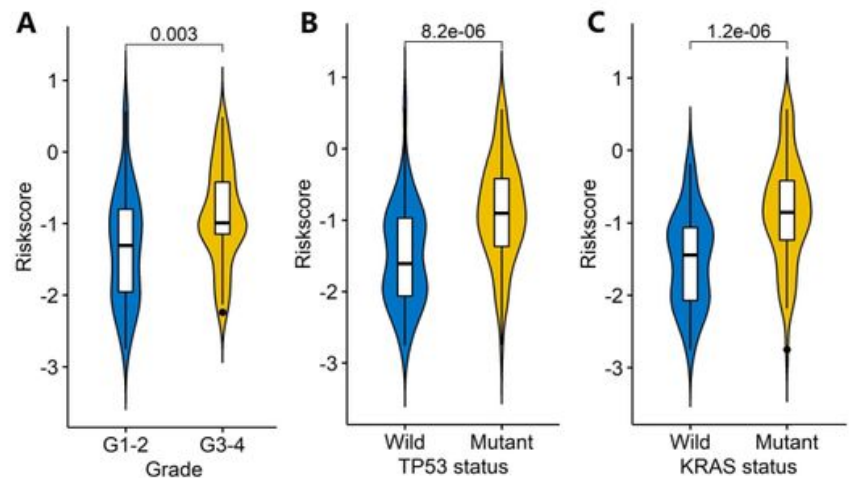

G
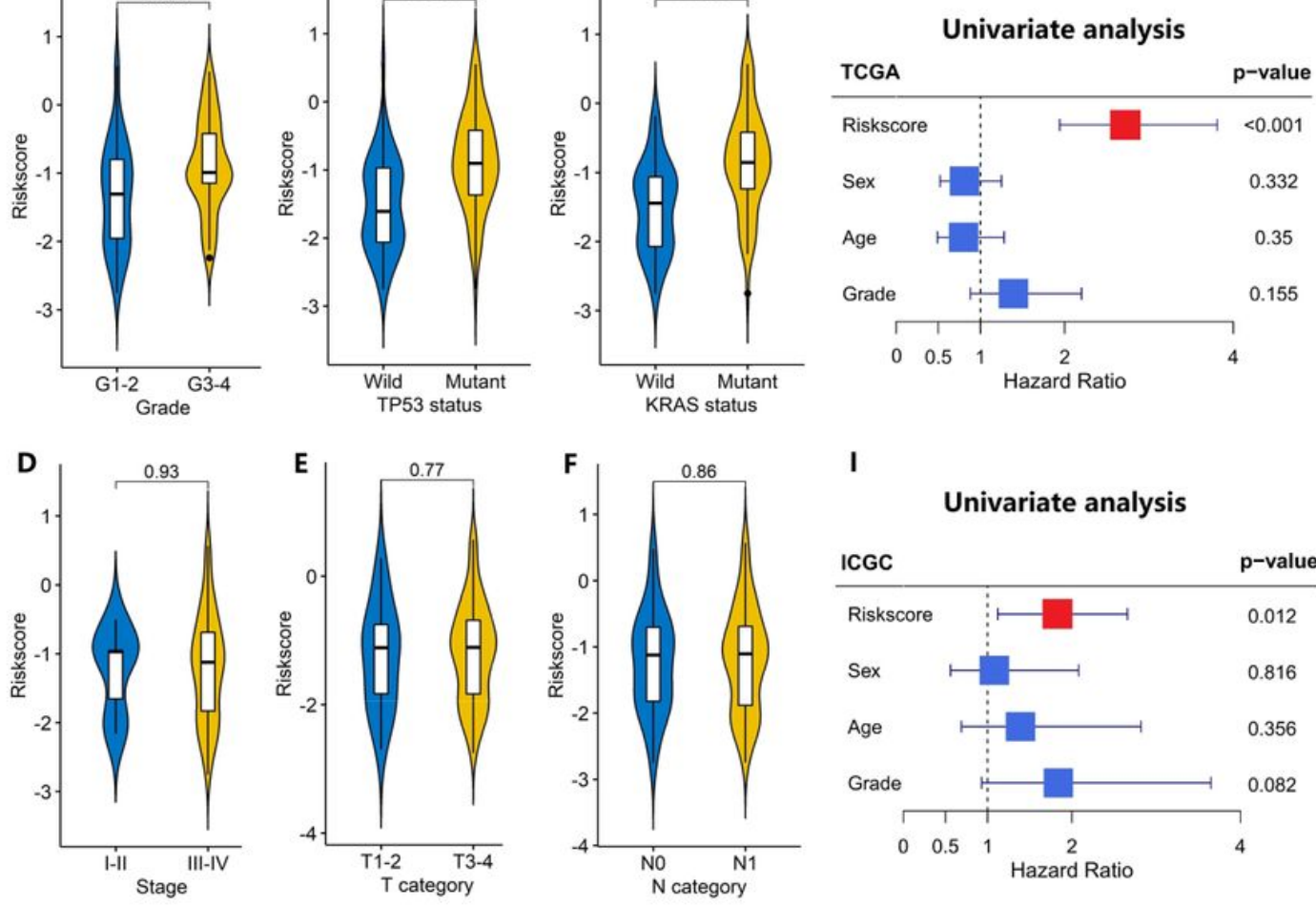

I

Univariate analysis

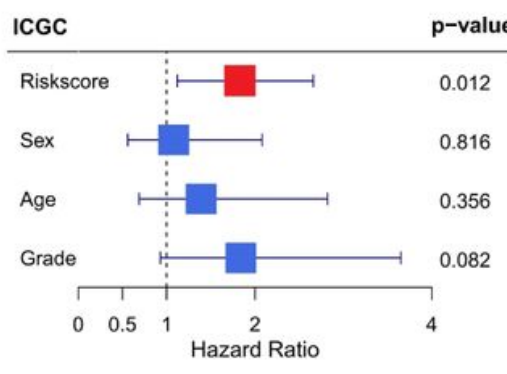

H

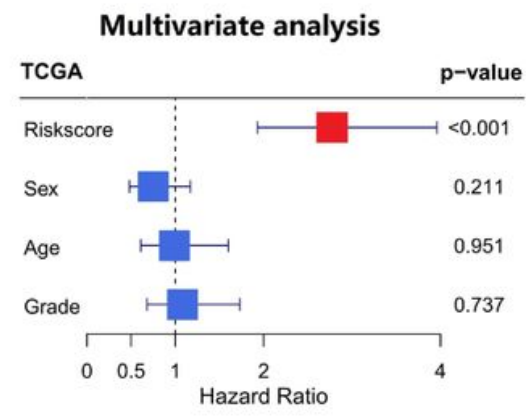

J

Multivariate analysis

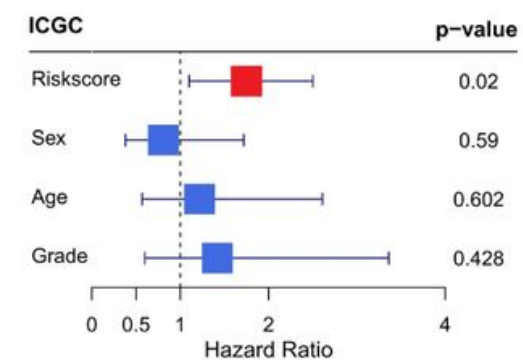

Figure 6

A-F Patients with different clinicopathological features (including grade, TP53 status and KRAS status, but not stage, $\mathrm{T}$ category and $\mathrm{N}$ category) had different levels of riskscore, calculated based on the m6A- 
related IncRNA prognostic riskscore (m6A-LPR). G-J Univariate and multivariate analyses revealed that riskscore was an independent prognostic predictor in the training $(\mathrm{G}-\mathrm{H})$ and validation $(\mathrm{I}-\mathrm{J})$ cohorts.
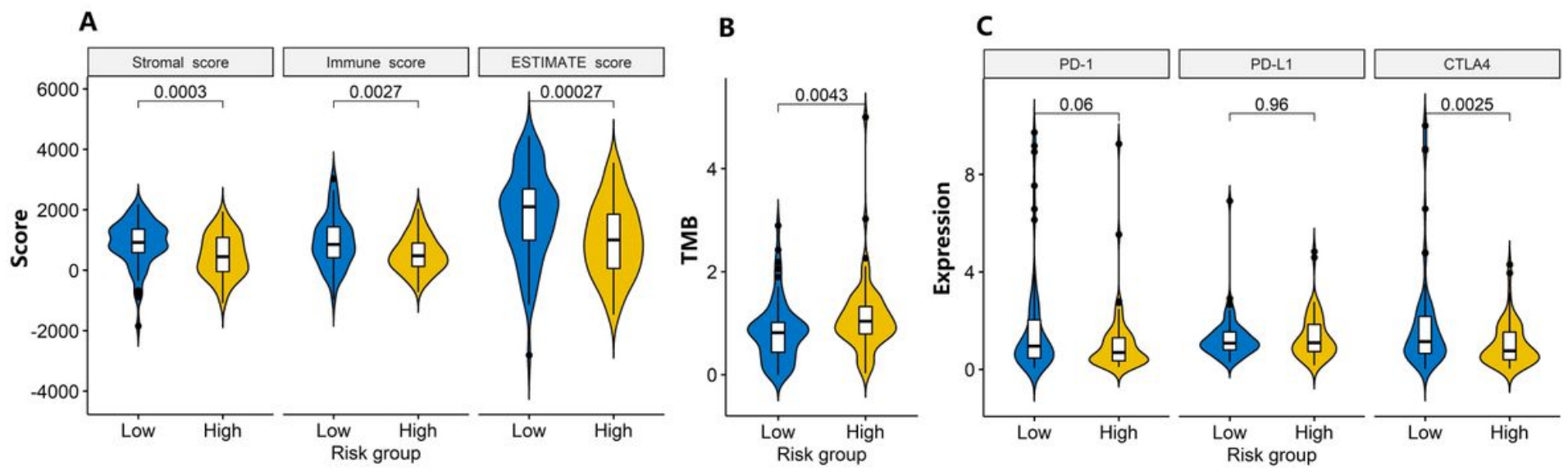

D

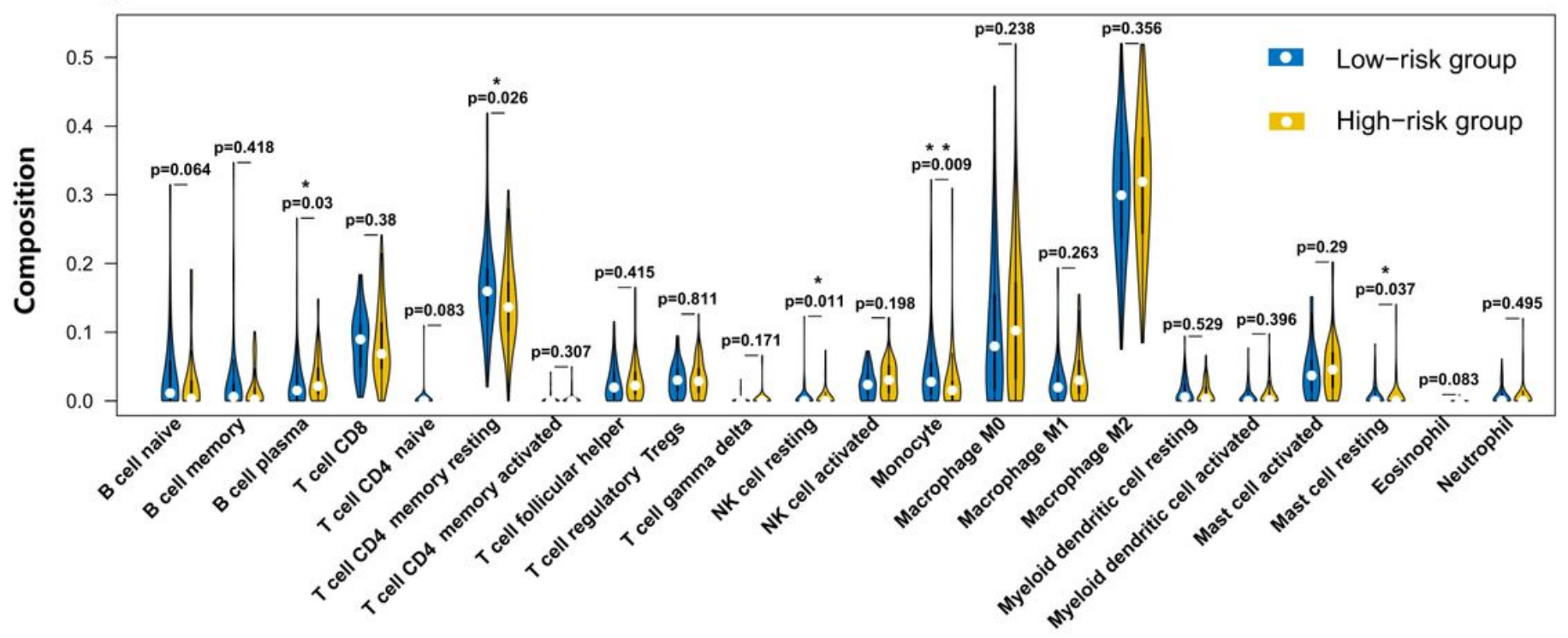

Figure 7

A-B The stromal score, immune score, tumor purity and tumor mutation burden (TMB) significantly differ between the low- and high-risk groups based on the m6A-related IncRNA prognostic riskscore (m6A-LPR) in the training cohort. C Comparison of the expression pattern of immune checkpoint genes (PD1, PD-L1, and CTLA-4) between the low- and high-risk groups based on the m6A-LPR in the training cohort. D Relative infiltrating proportion of immune cells in low- and high-risk groups. 


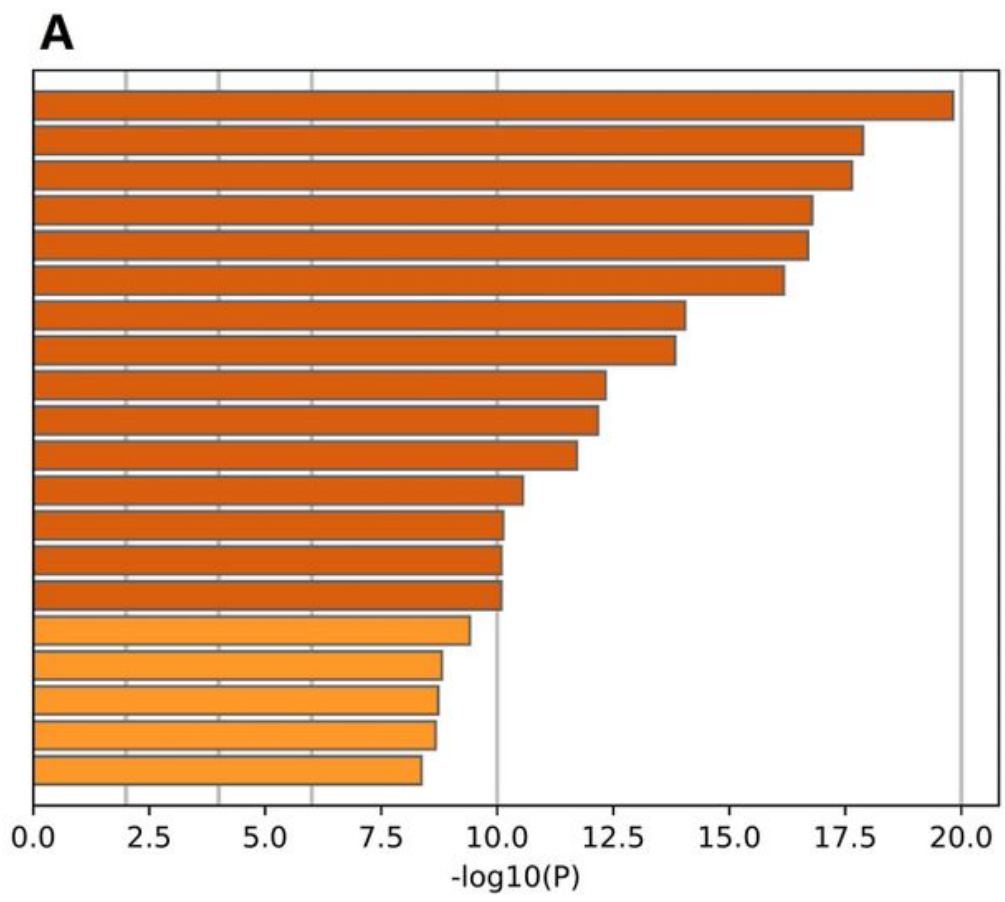

M5885: NABA MATRISOME ASSOCIATED GO:0043269: regulation of ion transport hsa04972: Pancreatic secretion GO:0007268: chemical synaptic transmission hsa 04080: Neuroactive ligand-receptor interaction GO:0044057: regulation of system process GO:0023061: signal release GO:0007586: digestion GO:0042391: regulation of membrane potential R-HSA-8935690: Digestion GO:0070268: cornification GO:0098771: inorganic ion homeostasis GO:0098662: inorganic cation transmembrane transport R-HSA-112316: Neuronal System R-HSA-2980736: Peptide hormone metabolism ko04060: Cytokine-cytokine receptor interaction GO:0007218: neuropeptide signaling pathway GO:0006820: anion transport GO:0019932: second-messenger-mediated signaling GO:0097305: response to alcohol

B

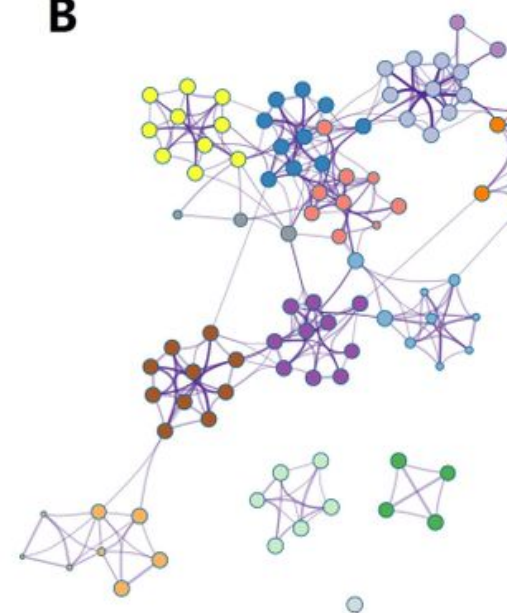

9
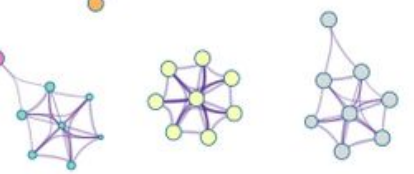

\section{Cluster ID}

NABA MATRISOME ASSOCIATED

regulation of ion transport

Pancreatic secretion

chemical synaptic transmission

Neuroactive ligand-receptor interaction regulation of system process

a signal release

digestion

regulation of membrane potential

Digestion

cornification

inorganic ion homeostasis

inorganic cation transmembrane transport

Neuronal System

Peptide hormone metabolism

Cytokine-cytokine receptor interaction neuropeptide signaling pathway

anion transport

second-messenger-mediated signaling

response to alcohol
C

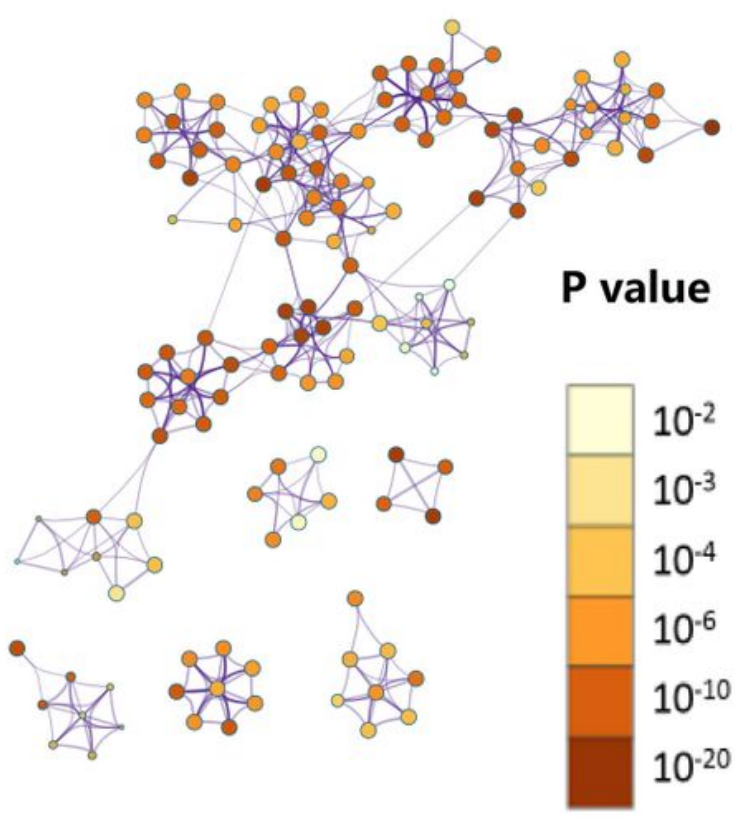

\section{Figure 8}

Functional analysis of 927 differentially expressed genes (DEGs) between the low- and high-risk groups. A Heatmap of enriched terms across the inputted gene list, colored according to $p$-value. Network of enriched terms colored according to B cluster ID (nodes with the same cluster ID are typically close to each other) and $\mathrm{C}$ p-value (terms with more genes tend to have higher $\mathrm{p}$-values). 


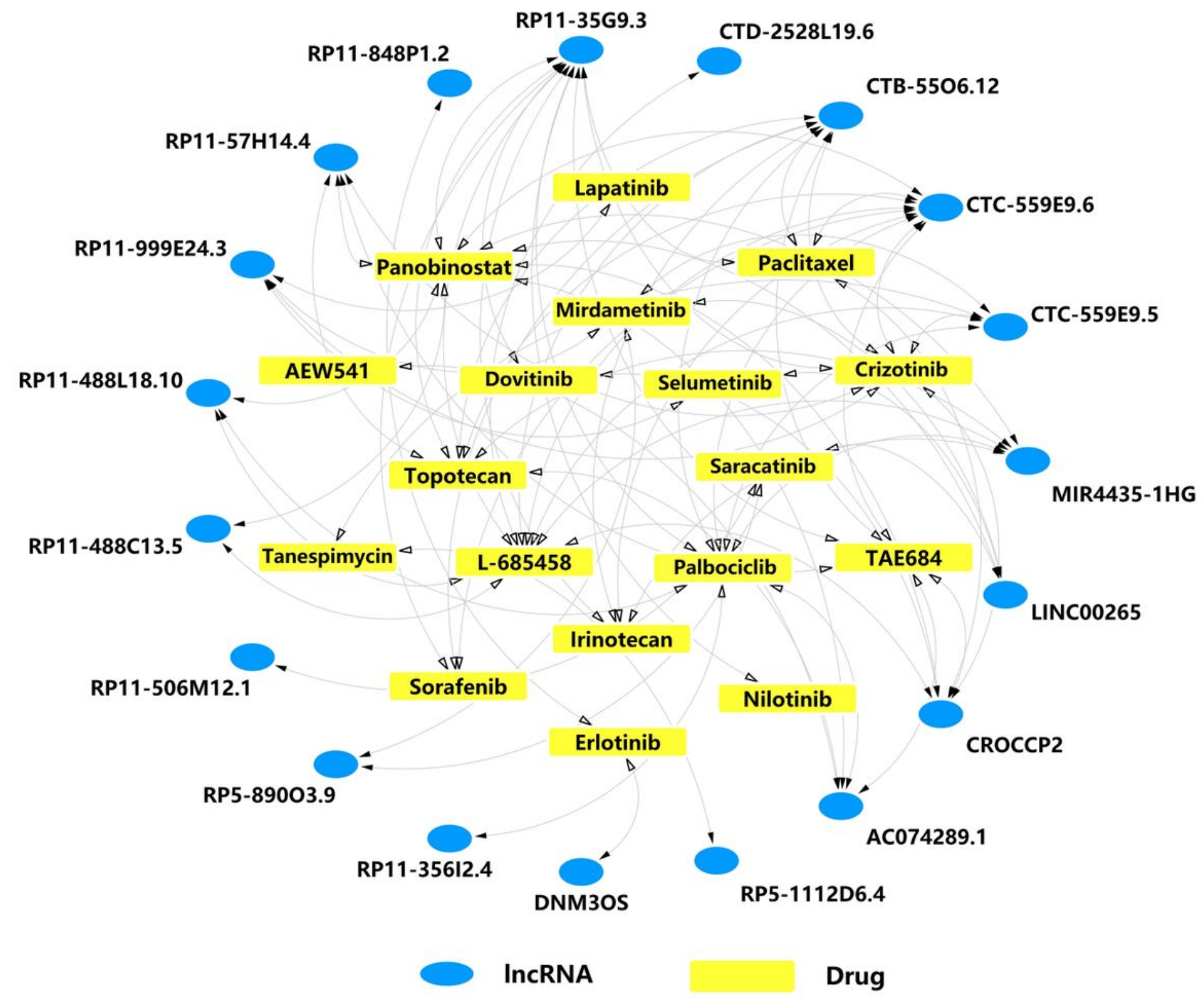

Figure 9

Potential targeted drugs for prognostic m6A-related IncRNAs in the Drug-LncRNA Module of the LncMAP database (False Discovery Rate (FDR) $<0.05$

\section{Supplementary Files}

This is a list of supplementary files associated with this preprint. Click to download.

- table1.xls

- Additionalfile1TableS1.xIsx 